\title{
Almost Contact Metric Structures on 5-Dimensional Nilpotent Lie Algebras
}

\author{
Nülifer Özdemir ${ }^{1,+}$, Mehmet Solgun ${ }^{2, *,+}$ and Şirin Aktay ${ }^{1,+}$ \\ 1 Department of Mathematics, Anadolu University, 26470 Eskişehir, Turkey; nozdemir@anadolu.edu.tr (N.Ö.); \\ sirins@anadolu.edu.tr (S..A.) \\ 2 Department of Mathematics, Bilecik Seyh Edebali University, 11210 Bilecik, Turkey \\ * Correspondence: mehmet.solgun@bilecik.edu.tr; Tel.: +90-506-244-04-11 \\ + These authors contributed equally to this work.
}

Academic Editor: Roman M. Cherniha

Received: 11 June 2016; Accepted: 28 July 2016; Published: 4 August 2016

\begin{abstract}
We study almost contact metric structures on 5-dimensional nilpotent Lie algebras and investigate the class of left invariant almost contact metric structures on corresponding Lie groups. We determine certain classes that a five-dimensional nilpotent Lie group can not be equipped with.
\end{abstract}

Keywords: 5-dimensional nilpotent Lie algebra; almost contact metric structure; left invariant almost contact metric structure

\section{Introduction}

It is well-known that every connected odd dimensional Lie group is equipped with a left invariant almost contact metric structure. These structures give rise to almost contact metric structures on corresponding Lie algebras [1]. In literature, some certain classes of such structures are studied. In [2], some general results on 5-dimensional Sasakian Lie algebras were stated, and it was proved that an odd dimensional nilpotent Lie group with a left invariant Sasakian structure is isomorphic to the real Heisenberg group. In addition, a classification of five-dimensional Sasakian Lie algebras were obtained. Then, in [3], left invariant K-contact structures on five-dimensional Lie groups were investigated. Three-dimensional homogeneous almost contact metric structures were considered in [4]. In [5], cosymplectic and $\alpha$-cosymplectic Lie algebras were investigated in terms of corresponding symplectic Lie algebras and suitable derivations on them.

Our aim in this manuscript is to determine almost contact metric structures on five-dimensional nilpotent Lie algebras by direct calculation. We use the classification of five-dimensional nilpotent Lie algebras given in [6]. We consider some certain classes of almost contact metric structures, and, by this approach, we get some general results on left invariant almost contact metric structures on five-dimensional nilpotent Lie groups.

\section{Preliminaries}

Let $M^{2 n+1}$ be a differentiable manifold of dimension $2 n+1$. If there is a $(1,1)$ tensor field $\phi$, a vector field $\xi$ and a one-form $\eta$ on $M$ satisfying:

$$
\phi^{2}=-I+\eta \otimes \xi, \quad \eta(\xi)=1,
$$

then, $M$ is said to have an almost contact structure $(\phi, \xi, \eta)$. A manifold with an almost contact structure is called an almost contact manifold. If, in addition to an almost contact structure $(\phi, \xi, \eta)$, $M$ also admits a Riemannian metric $g$ such that

$$
g(\phi(X), \phi(Y))=g(X, Y)-\eta(X) \eta(Y)
$$


for all vector fields $X, Y$, then $M$ is an almost contact metric manifold with the almost contact metric structure $(\phi, \xi, \eta, g)$. The Riemannian metric $g$ is called a compatible metric. The one-form defined by

$$
\Phi(X, Y)=g(X, \phi(Y))
$$

for all $X, Y \in \mathfrak{X}(M)$, is called the fundamental two-form of the almost contact metric manifold $(M, \phi, \xi, \eta, g)$. In [7], a classification of almost contact metric manifolds was obtained via the study of the covariant derivative of the fundamental two-form. A space having the same symmetries as the covariant derivative of the fundamental two-form was written, and, then, this space was decomposed into twelve $U(n) \times 1$ irreducible components $\mathcal{C}_{1}, \ldots, \mathcal{C}_{12}$. There are $2^{12}$ invariant subspaces, each corresponding to a class of almost contact metric manifolds. For example, the trivial class for which $\nabla \Phi=0$ [8], corresponds to the class of cosymplectic (called co-Kähler by some authors) manifolds, $\mathcal{C}_{1}$ is the class of nearly-K-cosymplectic manifolds, etc. [7]. For classification of almost contact metric structures (see also [9]). In this work, we focus on cosymplectic, nearly cosymplectic, $\alpha$-Sasakian, $\beta$-Kenmotsu and almost cosymplectic structures.

Let $(\phi, \xi, \eta, g)$ be an almost contact metric structure on $M$ with the fundamental two-form $\Phi$. $(\phi, \xi, \eta, g)$ is called:

- $\quad$ nearly cosymplectic if $\nabla_{X} \Phi(X, Y)=0$,

- $\quad \alpha$-Sasakian $\left(\mathcal{C}_{6}\right)$ if $\nabla_{X} \phi(Y)=\alpha(g(X, Y) \xi-\eta(Y) X)$ for a constant $\alpha$,

- $\quad \beta$-Kenmotsu $\left(\mathcal{C}_{5}\right)$ if $\nabla_{X} \phi(Y)=\beta(\Phi(X, Y) \eta(Z)-\Phi(X, Z) \eta(Y))$ for a constant $\beta$,

- $\quad$ semi cosymplectic $\left(\mathcal{C}_{1} \oplus \mathcal{C}_{2} \oplus \mathcal{C}_{3} \oplus \mathcal{C}_{7} \oplus \mathcal{C}_{8} \oplus \mathcal{C}_{9} \oplus \mathcal{C}_{10} \oplus \mathcal{C}_{11}\right)$ if $\delta \Phi=0$ and $\delta \eta=0$, where $\delta$ denotes the coderivative of a differential form,

- almost cosymplectic $\left(\mathcal{C}_{2} \oplus \mathcal{C}_{9}\right)$ if $d \Phi=0$ and $d \eta=0$, where $d$ denotes the exterior derivative of a differential form,

for all vector fields $X, Y, Z$ on $M$.

In literature, there are different but related definitions of cosymplectic structures. Here, we remind them and relate to the classes we use. In [5,10], an almost cosymplectic manifold is defined as a smooth manifold with a one-form $\eta$ and a two-form $\Phi$ such that $\eta \wedge \Phi^{n}$ is a volume form. If both $\eta$ and $\Phi$ are closed, then the manifold is said to be cosymplectic. In the same context, if $d \eta=0$ and $d \Phi=2 \alpha \eta \wedge \phi$ for a constant $\alpha$, then the manifold is called $\alpha$-cosymplectic. An almost contact metric manifold $(M, \phi, \xi, \eta, g)$, where $(\eta, \Phi)$ is a $\alpha$ cosymplectic structure is called an almost co-Kählbaser manifold. In addition, if this manifold is normal, then it is said to be co-Kähler. An almost contact metric manifold $(M, \phi, \xi, \eta, g)$ such that $(\eta, \Phi)$ is an $\alpha$-cosymplectic structure is called an almost $\alpha$ co-Kähler manifold. A normal almost $\alpha$ co-Kähler manifold is said to be $\alpha$ co-Kähler. Refer to $[5,10]$ and references therein. "Almost cosymplectic", "cosymlectic" and " $\alpha$-Kenmotsu" structures in our paper correspond to "almost co-Kähler", "co-Kähler" and " $\alpha$ co-Kähler" in [5], respectively. Throughout the paper, the definitions in and $[7,8]$ will be followed.

The existence of metric connections on five-dimensional almost contact metric manifolds compatible with the almost contact structure was investigated in [11]. The space of torsion tensors of a metric connection splits into ten $U(2)$-irreducible subspaces $\mathcal{W}_{1}, \mathcal{W}_{2}, \ldots, \mathcal{W}_{10}$. Thus, there are $2^{10}$ classes of almost contact metric structures in five-dimensions according to components of torsion tensor [11].

An almost contact metric structure $(\phi, \xi, \eta, g)$ on a connected Lie group $G$ is said to be left invariant if $g$ is left invariant and if the left multiplication map $L_{a}: G \longrightarrow G, L_{a}(x)=a \cdot x$ has properties

$$
\phi \circ L_{a}=L_{a} \circ \phi, \quad L_{a}(\xi)=\xi,
$$

for all $a \in G$. 
Let $g$ be an odd dimensional Lie algebra. An almost contact metric structure on $g$ is a quadruple $(\phi, \xi, \eta, g)$, where $\eta$ is a one-form, $\phi$ is an endomorphism of $g, \xi \in g$ such that

$$
\phi^{2}=-I+\eta \otimes \xi, \quad \eta(\xi)=1, \quad g(\phi(X), \phi(Y))=g(X, Y)-\eta(X) \eta(Y),
$$

for all vector fields $X, Y$ and $g$ is a positive definite compatible inner product on $g$. It is also convenient to use defining relations for the structures on Lie algebras. For instance, an almost contact metric structure $(\phi, \xi, \eta, g)$ on a Lie algebra $g$ is said to be nearly cosymplectic if $\nabla_{X} \Phi(X, Y)=0$ for any $X, Y$ in $g$, etc.

Let $G$ be a connected Lie group endowed with a left invariant almost contact metric structure $(\phi, \xi, \eta, g)$ and $g \cong T_{e} G$ be the corresponding Lie algebra of $G$. Then, this structure uniquely yields an almost contact metric structure $(\phi, \xi, \eta, g)$ on $g$.

In this work, we study almost contact metric structures on five-dimensional nilpotent Lie algebras. The classification of nilpotent Lie algebras of dimension $\leq 5$ was obtained in [6] (see also [12,13]). Indeed, $g_{i}$ are five-dimensional nilpotent algebras with the corresponding basis $\left\{e_{1}, \ldots, e_{5}\right\}$ and non-zero brackets as follows:

$$
\begin{aligned}
& g_{1}:\left[e_{1}, e_{2}\right]=e_{5},\left[e_{3}, e_{4}\right]=e_{5}, \\
& g_{2}:\left[e_{1}, e_{2}\right]=e_{3},\left[e_{1}, e_{3}\right]=e_{5},\left[e_{2}, e_{4}\right]=e_{5}, \\
& g_{3}:\left[e_{1}, e_{2}\right]=e_{3},\left[e_{1}, e_{3}\right]=e_{4},\left[e_{1}, e_{4}\right]=e_{5},\left[e_{2}, e_{3}\right]=e_{5}, \\
& g_{4}:\left[e_{1}, e_{2}\right]=e_{3},\left[e_{1}, e_{3}\right]=e_{4},\left[e_{1}, e_{4}\right]=e_{5}, \\
& g_{5}:\left[e_{1}, e_{2}\right]=e_{4},\left[e_{1}, e_{3}\right]=e_{5}, \\
& g_{6}:\left[e_{1}, e_{2}\right]=e_{3},\left[e_{1}, e_{3}\right]=e_{4},\left[e_{2}, e_{3}\right]=e_{5} .
\end{aligned}
$$

The rest of the classes $g_{7}, g_{8}, g_{9}$ are abelian.

\section{Almost Contact Metric Structures on $g_{i}$}

Let $G$ be a connected Lie group and $(\phi, \xi, \eta, g)$ a left invariant a.c.m.s. (almost contact metric structure) on $G$. Denote the corresponding a.c.m.s. on $g$ by the same symbols. Choose the basis $\left\{e_{1}, \ldots, e_{5}\right\}$ such that basis elements are $g$-orthonormal.

First, we investigate the existence of some classes of almost contact metric structures on each $g_{i}$.

The algebra $g_{1}$ : By Kozsul's formula, the covariant derivatives of the basis elements are as follows:

$$
\begin{array}{llll}
\nabla_{e_{1}} e_{2}=\frac{1}{2} e_{5}, & \nabla_{e_{1}} e_{5}=-\frac{1}{2} e_{2}, & \nabla_{e_{2}} e_{1}=-\frac{1}{2} e_{5}, & \nabla_{e_{2}} e_{5}=\frac{1}{2} e_{1}, \\
\nabla_{e_{3}} e_{4}=\frac{1}{2} e_{5}, & \nabla_{e_{3}} e_{5}=-\frac{1}{2} e_{4}, & \nabla_{e_{4}} e_{3}=-\frac{1}{2} e_{5}, & \nabla_{e_{4}} e_{5}=\frac{1}{2} e_{3}, \\
\nabla_{e_{5}} e_{1}=-\frac{1}{2} e_{2}, & \nabla_{e_{5}} e_{2}=\frac{1}{2} e_{1}, & \nabla_{e_{5} e_{3}}=-\frac{1}{2} e_{4}, & \nabla_{e_{5}} e_{4}=\frac{1}{2} e_{3} .
\end{array}
$$

- $\quad$ There exists no cosymplectic structure on $g_{1}$.

To see this, assume $\Phi=\sum b_{i j} e^{i j}$ is a two-form on $g_{1}$ such that $\nabla \Phi=0$. Then, for any elements $e_{i}, e_{j}, e_{k}$ of the basis:

$$
\begin{aligned}
\left(\nabla_{e_{i}} \Phi\right)\left(e_{j}, e_{k}\right) & =e_{i}\left[\Phi\left(e_{j}, e_{k}\right)\right]-\Phi\left(\nabla_{e_{i}} e_{j}, e_{k}\right)-\Phi\left(e_{j}, \nabla_{e_{i}} e_{k}\right) \\
& =-\Phi\left(\nabla_{e_{i}} e_{j}, e_{k}\right)-\Phi\left(e_{j}, \nabla_{e_{i}} e_{k}\right)=0 .
\end{aligned}
$$

It is easy to see that $\nabla \Phi=0$ if and only if $b_{i j}=0$ for any $i, j$. Thus, $(\phi, \xi, \eta, g)$ is not cosymplectic. 
- There is no nearly cosymplectic structure (i.e., $\left.\left(\nabla_{X} \Phi\right)(X, Y)=0\right)$. Let $\Phi=\sum b_{i j} e^{i j}$, by direct calculation, we obtain,

$$
\left(\nabla_{e_{i}} \Phi\right)\left(e_{i}, e_{j}\right)=0 \Longleftrightarrow b_{13} b_{23}+b_{14} b_{24}=b_{13} b_{14}+b_{23} b_{24}=0,
$$

where $b_{14}^{2}=b_{23}^{2}$ and $b_{13}^{2}=b_{24}^{2}$ and the remaining coefficents are zero. Thus $\Phi=b_{13} e^{13}+b_{14} e^{14}+$ $b_{23} e^{23}+b_{24} e^{24}$. By polarizing the equation $\left(\nabla_{X} \Phi\right)(X, Y)=0$, we get

$$
\nabla_{X} \Phi(Y, Z)+\nabla_{Y} \Phi(X, Z)=0 .
$$

Then for $X=e_{2}, Y=e_{3}$ and $Z=e_{5}$ in the equation (2), we obtain $b_{13}=-b_{24}$. In addition, replacing $e_{3}, e_{5}$ and $e_{2}$ for $X, Y, Z$ respectively in the equation (2), we get $b_{13}=2 b_{24}$. Thus, $b_{13}=b_{24}=0$. On the other hand, we get $b_{14}=b_{23}$ and $2 b_{23}=-b_{14}$ for $X=e_{3}, Y=e_{1}$ and $Z=e_{5}$ and $X=e_{1}, Y=e_{5}$ and $Z=e_{3}$ respectively in the equation (2), which implies $b_{14}=b_{23}=0$.

- There is no non-zero parallel vector field on $g_{1}$. Let $\xi=\sum a_{i} e_{i}$ be a parallel vector field on $g_{1}$ (i.e., $\nabla \xi=0$ ). Then, by the Kozsul formula, we have $a_{i}=0$ for $i=1, \ldots, 5$.

Note that since $\left(\nabla_{X} \eta\right)(Y)=g\left(\nabla_{X} \xi, Y\right)$ for all vector fields $X$ and $Y$, we have $\nabla \eta \neq 0$ for any almost contact metric structure $(\phi, \xi, \eta, g)$ on $g_{1}$. In particular, $(\phi, \xi, \eta, g)$ is neither $\mathcal{C}_{1}$ (nearly-K-cosymplectic), nor $\mathcal{C}_{2}$.

- A vector field $\xi$ on $g_{1}$ is Killing if and only if $\xi \in\left\langle e_{5}\right\rangle$.

Let $\xi=\sum a_{i} e_{i}$ be a Killing vector field. Then, for any $e_{i}, e_{j}$, we have $g\left(\nabla_{e_{i}} \xi, e_{j}\right)=-g\left(\nabla_{e_{j}} \xi_{,} e_{i}\right)$. Then,

$$
g\left(\nabla_{e_{2}} \xi, e_{5}\right)=-\frac{1}{2} a_{1} \text { and } g\left(\nabla_{e_{5}} \xi, e_{2}\right)=-\frac{1}{2} a_{1}
$$

yields $a_{1}=0$. Similarly, since $g\left(\nabla_{e_{1}} \xi, e_{5}\right)=-g\left(\nabla_{e_{5}} \xi, e_{1}\right)$, we have $a_{2}=0$ and $g\left(\nabla_{e_{4}} \xi, e_{5}\right)=-g\left(\nabla_{e_{5}} \xi, e_{4}\right)$ gives $a_{3}=0$. In addition, $g\left(\nabla_{e_{3}} \xi, e_{5}\right)=-g\left(\nabla_{e_{5}} \xi, e_{3}\right)$ implies $a_{4}=0$. As a result, $\xi=a_{5} e_{5}$.

- There exists $1 / 2$-Sasakian structure on $g_{1}$, where the fundamental two-form is $\Phi=-e^{12}-e^{34}$ and $\xi=e_{5}$. Note that this structure is given in [2] as a Sasakian structure because of the coefficient 2 in the defining relation of a Sasakian structure.

- $\quad$ There is no $\beta$-Kenmotsu structure.

Assume $(\phi, \xi, \eta, g)$ is a $\beta$-Kenmotsu structure with fundamental two-form $\Phi=\sum b_{i j} e^{i j}$. Then, $g\left(\nabla_{e_{i}} \xi, e_{j}\right)=g\left(\nabla_{e_{j}} \xi, e_{i}\right)$ for any basis elements $e_{i}, e_{j}$, which implies that $\xi=a_{1} e_{1}+a_{2} e_{2}+a_{3} e_{3}+$ $a_{4} e_{4}$ and $\eta=b_{1} e^{1}+b_{2} e^{2}+b_{3} e^{3}+b_{4} e^{4}$. On the other hand for $X=e_{1}, Y=e_{1}, Z=e_{2}$ and for $X=e_{1}, Y=e_{1}, Z=e_{5}$ in the defining relation of a $\beta$-Kenmotsu structure, we obtain $b_{15}=2 \beta b_{1} b_{12}$ and $b_{12}=-2 \beta b_{1} b_{15}$, respectively. Thus, $b_{12}=b_{15}=0$. Similar arguments work if $X, Y, Z$ are replaced by other basis elements. We get $b_{i j}=0$ for all $i, j$. As a result the structure is not $\beta$-Kenmotsu.

- $\quad$ There is no almost cosymplectic structure.

Let $\eta=\sum b_{i} e^{i}$ and $\Phi=\sum b_{i j} e^{i j}$. Then, since $d e^{5}=-e^{12}-e^{34}$ and $d e^{i}=0$ for $i=1,2,3,4$, we get $d \eta=-b_{5}\left(e^{12}+e^{34}\right)$. This yields $d \eta=0$ iff $b_{5}=0$. On the other hand, we have $d \Phi=b_{15} e^{134}+b_{25} e^{234}+b_{35} e^{123}+b_{45} e^{124}$, which is zero iff $b_{15}=b_{25}=b_{45}=0$. In this case, $\Phi \wedge \Phi=2\left(b_{12} b_{34}+b_{14} b_{23}-b_{13} b_{24}\right) e^{1234}$ and $\eta \wedge \Phi^{2}=0$, which contradicts with the assumption that $(\phi, \xi, \eta, g)$ is an almost contact metric structure.

- There are semi cosymplectic structures on $g_{1}$.

For any vector $X=\sum x_{i} e_{i}$ on $g_{1}$, we have $\delta \Phi(X)=x_{5}\left(b_{12}+b_{34}\right)$. Thus, $\delta \Phi=0$ for all $X$ iff $b_{12}=-b_{34}$. In addition, $\delta \eta=0$ for any one-form $\eta$. Choose, for example, the a.c.m.s. $(\phi, \xi, \eta, g)$, where $\xi=e_{5}, \eta=e^{5}$ and $\Phi=e^{12}-e^{34}$. This structure is semi cosymplectic.

- Consider the a.c.m.s. $(\phi, \xi, \eta, g)$, where $\phi\left(e_{1}\right)=-e_{4}, \phi\left(e_{2}\right)=-e_{3}, \phi\left(e_{3}\right)=e_{2}, \phi\left(e_{4}\right)=e_{1}$ and $\xi=e_{5}, \eta=e^{5}$ on $g_{1}$. We show that there is a metric connection $\nabla^{c}$ compatible with this structure. 
Assume that $\nabla^{c}$ is a metric connection of $g$. Then, $\nabla^{c}=\nabla+A$, where $A$ is a skew-symmetric $(2,1)$ tensor field. Since $\nabla^{c}$ is compatible with $\xi=e_{5}$, we have $\nabla_{e_{i}}^{c_{e}} e_{5}=0$ for all basis elements $e_{i}$. We obtain

$$
A\left(e_{1}, e_{5}\right)=\frac{1}{2} e_{2}, A\left(e_{2}, e_{5}\right)=-\frac{1}{2} e_{1}, A\left(e_{3}, e_{5}\right)=\frac{1}{2} e_{4}, A\left(e_{4}, e_{5}\right)=-\frac{1}{2} e_{3} .
$$

Metric compatibility of $\nabla^{c}$ yields

$$
0=e_{1}\left[g\left(e_{1}, e_{2}\right)\right]=g\left(\nabla_{e_{1}}^{c} e_{1}, e_{2}\right)+g\left(e_{1}, \nabla_{e_{1}}^{c} e_{2}\right),
$$

and thus $g\left(e_{1}, \nabla_{e_{1}}^{c} e_{2}\right)=0$. Note that $\nabla_{e_{1}}^{c} e_{1}=\nabla_{e_{1}} e_{1}+A\left(e_{1}, e_{1}\right)=0$. Similarly, $g\left(e_{2}, \nabla_{e_{1}}^{c} e_{2}\right)=$ $g\left(e_{5}, \nabla_{e_{1}}^{c} e_{2}\right)=0$. Hence, $\nabla_{e_{1}}^{c} e_{2}=a_{3} e_{3}+a_{4} e_{4}$ for some constants $a_{3}, a_{4}$ and $A\left(e_{1}, e_{2}\right)=a_{3} e_{3}+$ $a_{4} e_{4}-\frac{1}{2} e_{5}$.

Since $\nabla^{c}$ is also compatible with $\phi$, that is, $\nabla^{c} \phi=0$, we have

$$
0=\left(\nabla_{e_{1}}^{c} \phi\right)\left(e_{2}\right)=\nabla_{e_{1}}^{c}\left(\phi\left(e_{2}\right)\right)-\phi\left(\nabla_{e_{1}}^{c} e_{2}\right)=-\nabla_{e_{1}}^{c} e_{3}-\phi\left(a_{3} e_{3}+a_{4} e_{4}\right) .
$$

Thus,

$$
\nabla_{e_{1}}^{c} e_{3}=-a_{3} e_{2}-a_{4} e_{1}=A\left(e_{1}, e_{3}\right) .
$$

In addition, $\left(\nabla_{e_{1}}^{c} \phi\right)\left(e_{4}\right)=0$ implies $\phi\left(\nabla_{e_{1}}^{c} e_{4}\right)=0$. By the identity $\phi^{2}=-I+\eta \otimes \xi$, we get

$$
0=\phi^{2}\left(\nabla_{e_{1}}^{c} e_{4}\right)=-\nabla_{e_{1}}^{c} e_{4}+g\left(\nabla_{e_{1}}^{c} e_{4}, e_{5}\right) e_{5},
$$

which gives $\nabla_{e_{1}}^{c} e_{4}=A\left(e_{1}, e_{4}\right)=0$. Note that $g\left(\nabla_{e_{1}}^{c} e_{4}, e_{5}\right)=0$ since $\nabla^{c}$ is a metric connection. Similarly, $\nabla_{e_{2}}^{c} e_{3}=A\left(e_{2}, e_{3}\right)=0$. By direct calculation, we get

$$
\begin{gathered}
\nabla_{e_{2}}^{c} e_{4}=A\left(e_{2}, e_{4}\right)=a_{4} e_{1}+a_{3} e_{2}, \\
\nabla_{e_{3}}^{c} e_{4}=a_{3} e_{3}+a_{4} e_{4}, A\left(e_{3}, e_{4}\right)=a_{3} e_{3}+a_{4} e_{4}-\frac{1}{2} e_{5} .
\end{gathered}
$$

To sum up,

$$
\begin{aligned}
A= & e_{1} \otimes\left\{a_{4}\left(-e^{13}+e^{24}\right)-\frac{1}{2} e^{25}\right\}+e_{2} \otimes\left\{a_{3}\left(-e^{13}+e^{24}\right)+\frac{1}{2} e^{15}\right\} \\
& +e_{3} \otimes\left\{a_{3} e^{12}-\frac{1}{2} e^{45}\right\}+e_{4} \otimes\left\{a_{4} e^{12}+\frac{1}{2} e^{35}\right\}+e_{5} \otimes\left\{-\frac{1}{2} e^{12}-\frac{1}{2} e^{34}\right\} .
\end{aligned}
$$

Since $(\phi, \xi, \eta, g)$ has a totally skew-symmetric metric connection, by Proposition 4.1 in [11], we conclude that $(\phi, \xi, \eta, g)$ is in the class $\mathcal{W}_{3} \oplus \mathcal{W}_{4} \oplus \mathcal{W}_{5} \oplus \mathcal{W}_{6}$ with respect to the classification of Puhle in [11].

Similar observations can be made for existing structures on each $g_{i}$.

The algebra $g_{2}$ : By Kozsul's formula, the covariant derivatives of the basis elements are as follows:

$$
\begin{array}{llll}
\nabla_{e_{1}} e_{2}=\frac{1}{2} e_{3}, & \nabla_{e_{1} e_{3}}=-\frac{1}{2} e_{2}+\frac{1}{2} e_{5}, & \nabla_{e_{1}} e_{5}=-\frac{1}{2} e_{3}, & \nabla_{e_{2}} e_{1}=-\frac{1}{2} e_{3}, \\
\nabla_{e_{2}} e_{3}=\frac{1}{2} e_{1}, & \nabla_{e_{2} e_{4}}=\frac{1}{2} e_{5}, & \nabla_{e_{2}} e_{5}=-\frac{1}{2} e_{4}, & \nabla_{e_{3} e_{1}}=-\frac{1}{2} e_{2}-\frac{1}{2} e_{5}, \\
\nabla_{e_{3}} e_{2}=\frac{1}{2} e_{1}, & \nabla_{e_{3}} e_{5}=\frac{1}{2} e_{1}, & \nabla_{e_{4}} e_{2}=-\frac{1}{2} e_{5}, & \nabla_{e_{4} e_{5}}=\frac{1}{2} e_{2}, \\
\nabla_{e_{5}} e_{1}=-\frac{1}{2} e_{3}, & \nabla_{e_{5} e_{2}}=-\frac{1}{2} e_{4}, & \nabla_{e_{5}} e_{3}=\frac{1}{2} e_{1}, & \nabla_{e_{5} e_{4}}=\frac{1}{2} e_{2},
\end{array}
$$


- There exists no cosymplectic structure.

The proof is similar to that of $g_{1}$.

- There exists no nearly cosymplectic structure.

Assume that there exists a nearly cosymplectic structure $(\phi, \xi, \eta, g)$ with the fundamental two-form $\Phi=\sum b_{i j} e^{i j}$. Then, for any basis elements $e_{i}, e_{j}$, we have $\left(\nabla_{e_{i}} \Phi\right)\left(e_{i}, e_{j}\right)=-\Phi\left(e_{i}, \nabla_{e_{i}} e_{j}\right)=0$. Thus, we get:

$\left(\nabla_{e_{1}} \Phi\right)\left(e_{1}, e_{2}\right)=0 \Rightarrow b_{13}=0,\left(\nabla_{e_{1}} \Phi\right)\left(e_{1}, e_{3}\right)=0 \Rightarrow b_{12}=b_{15},\left(\nabla_{e_{2}} \Phi\right)\left(e_{2}, e_{1}\right)=0 \Rightarrow b_{23}=0$, $\left(\nabla_{e_{2}} \Phi\right)\left(e_{2}, e_{3}\right)=0 \Rightarrow b_{12}=0$,

$\left(\nabla_{e_{2}} \Phi\right)\left(e_{2}, e_{4}\right)=0 \Rightarrow b_{25}=0,\left(\nabla_{e_{2}} \Phi\right)\left(e_{2}, e_{5}\right)=0 \Rightarrow b_{24}=0$,

$\left(\nabla_{e_{3}} \Phi\right)\left(e_{3}, e_{1}\right)=0 \Rightarrow b_{35}=0$ and $\left(\nabla_{e_{4}} \Phi\right)\left(e_{4}, e_{2}\right)=0 \Rightarrow b_{45}=0$.

Thus, the fundamental two-form is of type $\Phi=b_{14} e^{14}+b_{34} e^{34}$. From the equation $\Phi(X, Y)=g(X, \phi(Y))$, the endomorphism $\phi$ is defined by $\phi\left(e_{1}\right)=-b_{14} e_{4}, \phi\left(e_{2}\right)=0$, $\phi\left(e_{3}\right)=-b_{34} e_{4}, \phi\left(e_{4}\right)=b_{14} e_{1}+b_{34} e_{3}, \phi\left(e_{5}\right)=0$. Let $\xi=\sum_{i=1}^{5} a_{i} e_{i}$ and $\eta=\sum_{i=1}^{5} b_{i} e^{i}$. Then,

$\phi^{2}\left(e_{2}\right)=0=-e_{2}+\eta\left(e_{2}\right) \xi \Rightarrow b_{2} a_{2}=1, b_{2} a_{5}=0 \Rightarrow a_{5}=0$.

On the other hand,

$\phi^{2}\left(e_{5}\right)=0=-e_{5}+\eta\left(e_{5}\right) \Rightarrow b_{5} a_{5}=1 \Rightarrow a_{5} \neq 0$. Therefore, the condition $\phi^{2}=-I+\eta \otimes \xi$ does not hold. Thus, the structure is not nearly cosymplectic.

- $\quad$ There is no non-zero parallel vector field on $g_{2}$.

If a non-zero vector field $\xi=\sum a_{i} e_{i}$ is parallel $(\nabla \xi=0)$, by calculating $g\left(\nabla_{e_{i}} \xi, e_{j}\right)$ for basis elements, we get $a_{i}=0$, for $i=1, \cdots, 5$. It also shows that $\nabla \eta \neq 0$ for any almost contact metric structure $(\phi, \xi, \eta, g)$ on $g_{2}$. In particular, $(\phi, \xi, \eta, g)$ is neither $\mathcal{C}_{1}$ (nearly-K-cosymplectic), nor $\mathcal{C}_{2}$.

- A vector field $\xi$ on $g_{2}$ is Killing if and only if $\xi \in\left\langle e_{5}\right\rangle$.

Assume $\xi=\sum a_{i} e_{i}$ is a Killing vector field. Then, for any $e_{i}, e_{j}$, we have $g\left(\nabla_{e_{i}} \xi, e_{j}\right)=-g\left(\nabla_{e_{j}} \xi, e_{i}\right)$. Thus,

$$
g\left(\nabla_{e_{2}} \xi, e_{3}\right)=-\frac{1}{2} a_{1}, g\left(\nabla_{e_{3}} \xi, e_{2}\right)=-\frac{1}{2} a_{1} \Rightarrow a_{1}=0,
$$

and similarly,

$$
\begin{aligned}
& g\left(\nabla_{e_{4}} \xi, e_{5}\right)=-g\left(\nabla_{e_{5}} \xi, e_{4}\right) \Rightarrow a_{2}=0, \\
& g\left(\nabla_{e_{1}} \xi, e_{5}\right)=-g\left(\nabla_{e_{5}} \xi, e_{1}\right) \Rightarrow a_{3}=0, \\
& g\left(\nabla_{e_{2}} \xi, e_{5}\right)=-g\left(\nabla_{e_{5}} \xi, e_{2}\right) \Rightarrow a_{4}=0 .
\end{aligned}
$$

- There is no $\alpha$-Sasakian structure. Assume that a structure $(\phi, \xi, \eta, g)$ on $g_{2}$ is $\alpha$-Sasakian. Then, $\xi \in\left\langle e_{5}\right\rangle$, since it is a Killing vector field. On the other hand, by considering the relation $\nabla_{X} \xi=-\alpha \phi(X)$, we get the endomorphism:

$$
\phi\left(e_{1}\right)=\frac{a_{5}}{2 \alpha} e_{3}, \phi\left(e_{2}\right)=\frac{a_{5}}{2 \alpha} e_{4}, \phi\left(e_{3}\right)=-\frac{a_{5}}{2 \alpha} e_{1}, \phi\left(e_{4}\right)=-\frac{a_{5}}{2 \alpha} e_{2} .
$$

In addition, the structure must satisfy the defining relation of the class of $\alpha$-Sasakian structures:

$$
\left(\nabla_{X} \phi\right)(Y)=\alpha(g(X, Y) \xi-\eta(Y) X) .
$$

However, it is easy to see that this relation is not satisfied for $X=Y=e_{1}$. Hence, the structure is not $\alpha$-Sasakian.

Let $(\phi, \xi, \eta, g)$ is a $\beta$-Kenmotsu structure with fundamental two-form $\Phi=\sum b_{i j} e^{i j}$. Then, $g\left(\nabla_{e_{i}} \xi, e_{j}\right)=g\left(\nabla_{e_{j}} \xi, e_{i}\right)$ for any basis elements $e_{i}, e_{j}$. Since $g\left(\nabla_{e_{1}} \xi, e_{2}\right)=-\frac{a_{3}}{2}, g\left(\nabla_{e_{2}} \xi, e_{1}\right)=$ $\frac{a_{3}}{2} \Rightarrow a_{3}=0$ and $g\left(\nabla_{e_{2}} \xi, e_{4}\right)=-\frac{a_{5}}{2}, g\left(\nabla_{e_{4}} \xi, e_{2}\right)=\frac{a_{5}}{2} \Rightarrow a_{4}=0$, we have $\xi=a_{1} e_{1}+a_{2} e_{2}+a_{4} e_{4}$ and $\eta=b_{1} e^{1}+b_{2} e^{2}+b_{4} e^{4}$. On the other hand, for $X=e_{1}, Y=e_{3}, Z=e_{5}$ and for $X=e_{3}$, $Y=e_{3}, Z=e_{5}$ in the defining relation of a $\beta$-Kenmotsu structure, we obtain $b_{25}=0$ and $b_{13}=0$, 
respectively. Similar arguments work if $X, Y, Z$ are replaced by other basis elements. Thus, we get $b_{i j}=0$ for all $i, j$. As a result, the structure is not $\beta$-Kenmotsu.

- There exists a semi cosymplectic structure.

By checking covariant derivatives, it can be seen that $\delta \eta=0$ for any one-form $\eta$. In addition, for a two-form $\Phi=\sum b_{i j} e^{i j}$, by assuming $\delta \Phi=0$, we get $b_{12}=0$ and $b_{13}=-b_{24}$. If we choose $\Phi=e^{13}-e^{24}$, then the endomorphism $\phi$ is $\phi\left(e_{1}\right)=-e_{3}, \phi\left(e_{2}\right)=e_{4}, \phi\left(e_{3}\right)=e_{1}$, $\phi\left(e_{4}\right)=-e_{2}, \phi\left(e_{5}\right)=0$. For $\xi=e_{5}$ and $\eta=e^{5},(\phi, \xi, \eta, g)$ is a semi cosymplectic structure on $g_{2}$.

- There exists an almost cosymplectic structure.

The almost contact metric structure $(\phi, \xi, \eta, g)$, where $\xi=e_{2}, \eta=e^{2}$ and $\Phi=e^{15}+e^{34}$ is almost cosymplectic, that is $d \Phi=d \eta=0$.

The algebra $g_{3}$ : By Kozsul's formula, the covariant derivatives of the basis elements are as follows:

$$
\begin{array}{llll}
\nabla_{e_{1}} e_{2}=\frac{1}{2} e_{3}, & \nabla_{e_{1}} e_{3}=-\frac{1}{2} e_{2}+\frac{1}{2} e_{4}, & \nabla_{e_{1}} e_{4}=-\frac{1}{2} e_{3}+\frac{1}{2} e_{5}, & \nabla_{e_{1}} e_{5}=-\frac{1}{2} e_{4}, \\
\nabla_{e_{2}} e_{1}=-\frac{1}{2} e_{3}, & \nabla_{e_{2}} e_{3}=\frac{1}{2} e_{1}+\frac{1}{2} e_{5}, & \nabla_{e_{2}} e_{5}=-\frac{1}{2} e_{3}, & \\
\nabla_{e_{3}} e_{1}=-\frac{1}{2} e_{2}-\frac{1}{2} e_{4}, & \nabla_{e_{3}} e_{2}=\frac{1}{2} e_{1}-\frac{1}{2} e_{5}, & \nabla_{e_{3}} e_{4}=\frac{1}{2} e_{1}, & \nabla_{e_{3} e_{5}=\frac{1}{2} e_{2},} \\
\nabla_{e_{4} e_{1}}=-\frac{1}{2} e_{3}-\frac{1}{2} e_{5}, & \nabla_{e_{4}} e_{3}=\frac{1}{2} e_{1}, & \nabla_{e_{4}} e_{5}=\frac{1}{2} e_{1}, & \\
\nabla_{e_{5}} e_{1}=-\frac{1}{2} e_{4}, & \nabla_{e_{5}} e_{2}=-\frac{1}{2} e_{3}, & \nabla_{e_{5}} e_{3}=\frac{1}{2} e_{2}, & \nabla_{e_{5} e_{4}}=\frac{1}{2} e_{1} .
\end{array}
$$

- There exists no cosymplectic structure.

The proof is similar to that of $g_{1}$.

- There exists no nearly cosymplectic structure.

Let $(\phi, \xi, \eta, g)$ be a nearly cosymplectic structure with fundamental two-form $\Phi=\sum b_{i j} e^{i j}$. Then, for any basis elements $e_{i}, e_{j}$, we have $\left(\nabla_{e_{i}} \Phi\right)\left(e_{i}, e_{j}\right)=-\Phi\left(e_{i}, \nabla_{e_{i}} e_{j}\right)=0$. After some calculations, we see that this equation holds if and only if $\Phi=b_{24} e^{24}$. However, the condition $\eta \wedge \Phi \wedge \Phi \neq 0$ is not satisfied since $\Phi \wedge \Phi=0$.

- $\quad$ There is no non-zero parallel vector field on $g_{3}$.

The proof is similar to these of $g_{1}$ and $g_{2}$.

- A vector field $\xi$ on $g_{3}$ is Killing if and only if $\xi \in\left\langle e_{5}\right\rangle$.

Let $\xi=\sum a_{i} e_{i}$ be a Killing vector field. Then, for any $e_{i}, e_{j}$, we have $g\left(\nabla_{e_{i}} \xi, e_{j}\right)=-g\left(\nabla_{e_{j}} \xi, e_{i}\right)$. Now, $g\left(\nabla_{e_{1}} \xi, e_{3}\right)=-g\left(\nabla_{e_{3}} \xi, e_{1}\right) \Rightarrow a_{2}=0$,

$g\left(\nabla_{e_{1}} \xi, e_{4}\right)=-g\left(\nabla_{e_{4}} \xi, e_{1}\right) \Rightarrow a_{3}=0$,

$g\left(\nabla_{e_{1}} \xi, e_{5}\right)=-g\left(\nabla_{e_{5}} \xi, e_{1}\right) \Rightarrow a_{4}=0$,

$g\left(\nabla_{e_{2}} \xi, e_{3}\right)=-g\left(\nabla_{e_{3}} \xi, e_{2}\right) \Rightarrow a_{1}=0$. In other words, $\xi$ is Killing if and only if $\xi=a_{5} e_{5}$

- There is no $\alpha$-Sasakian structure.

Let $(\phi, \xi, \eta, g)$ be an $\alpha$-Sasakian structure on $g_{3}$. Then, $\xi \in\left\langle e_{5}\right\rangle$, since it is a Killing vector field. On the other hand, by considering the relation $\nabla_{X} \xi=-\alpha \phi(X)$, we get the endomorphism as:

$$
\phi\left(e_{1}\right)=\frac{a_{5}}{2 \alpha} e_{4}, \phi\left(e_{2}\right)=\frac{a_{5}}{2 \alpha} e_{3}, \phi\left(e_{3}\right)=-\frac{a_{5}}{2 \alpha} e_{2}, \phi\left(e_{4}\right)=-\frac{a_{5}}{2 \alpha} e_{1} .
$$

However, for $X=Y=e_{1}$, this structure does not satisfy the the defining relation $\left(\nabla_{X} \phi\right)(Y)=\alpha(g(X, Y) \xi-\eta(Y) X)$.

- There is no $\beta$-Kenmotsu structure.

Let $(\phi, \xi, \eta, g)$ be a $\beta$-Kenmotsu structure with fundamental two-form $\Phi=\sum b_{i j} e^{i j}, \xi=\sum a_{i} e_{i}$, $\eta=\sum b_{i} e^{i}$. Then, $g\left(\nabla_{e_{i}} \xi, e_{j}\right)=g\left(\nabla_{e_{j}} \xi, e_{i}\right)$ for any basis elements $e_{i}, e_{j}$, which implies that $\xi=a_{1} e_{1}+a_{2} e_{2}$ and $\eta=b_{1} e^{1}+b_{2} e^{2}$. However, replacing basis elements for vector fields in the 
defining relation of a $\beta$-Kenmotsu structure, we get $b_{i j}=0$, for any $i, j$. Thus, there does not exist a $\beta$-Kenmotsu structure.

- There exists a semi cosymplectic structure.

The almost contact metric structure $(\phi, \xi, \eta, g)$ for which $\xi=e_{5}, \eta=e^{5}$ and $\Phi=e^{14}-e^{23}$ is semi cosymplectic, that is, $\delta \Phi=\delta \eta=0$.

- There exists an almost cosymplectic structure.

The almost contact metric structure $(\phi, \xi, \eta, g)$, such that $\xi=e_{1}, \eta=e^{1}$ and $\Phi=e^{25}-e^{34}$ is almost cosymplectic.

The algebra $g_{4}$ : By Kozsul's formula, the covariant derivatives of the basis elements are as follows:

$$
\begin{gathered}
\nabla_{e_{1}} e_{2}=\frac{1}{2} e_{3}, \nabla_{e_{1}} e_{3}=-\frac{1}{2} e_{2}+\frac{1}{2} e_{4}, \nabla_{e_{1}} e_{4}=-\frac{1}{2} e_{3}+\frac{1}{2} e_{5}, \nabla_{e_{1}} e_{5}=-\frac{1}{2} e_{4}, \\
\nabla_{e_{2}} e_{1}=-\frac{1}{2} e_{3}, \quad \nabla_{e_{2}} e_{3}=\frac{1}{2} e_{1}, \\
\nabla_{e_{3}} e_{1}=-\frac{1}{2} e_{2}-\frac{1}{2} e_{4}, \nabla_{e_{3}} e_{2}=\frac{1}{2} e_{1}, \nabla_{e_{3}} e_{4}=\frac{1}{2} e_{1}, \\
\nabla_{e_{4}} e_{1}=-\frac{1}{2} e_{3}-\frac{1}{2} e_{5}, \nabla_{e_{4}} e_{3}=\frac{1}{2} e_{1}, \nabla_{e_{4}} e_{5}=\frac{1}{2} e_{1}, \\
\nabla_{e_{5}} e_{1}=-\frac{1}{2} e_{4}, \quad \nabla_{e_{5}} e_{4}=\frac{1}{2} e_{1} .
\end{gathered}
$$

- There exists no cosymplectic structure.

Assume that the two-form $\Phi=\sum b_{i j} e^{i j}$ is parallel. Then, $\left(\nabla_{e_{i}} \Phi\right)\left(e_{j}, e_{k}\right)=0$ for any basis elements $e_{i}, e_{j}, e_{k}$. This gives $b_{i j}=0$. Thus, there is no non-zero parallel two-form on $g_{4}$.

- $\quad$ There is no nearly cosymplectic structure on $g_{4}$.

Let $\Phi=\sum b_{i j} e^{i j}$ be the two-form of a nearly cosymplectic a.c.m.s. Replacing $X$ and $Y$ by basis elements, we have $\left(\nabla_{e_{1}} \Phi\right)\left(e_{1}, e_{2}\right)=-\frac{1}{2} b_{13}=0$ and similarly $b_{i j}=0$, except for $b_{24}, b_{25}$ and $b_{35}$. Thus, $\Phi=b_{24} e^{24}+b_{25} e^{25}+b_{35} e^{35}$. We get $b_{24}=0, b_{25}=0$ and $b_{35}=0$ for $X=e_{1}, Y=e_{2}, Z=e_{3}$; $X=e_{1}, Y=e_{2}, Z=e_{4}$ and $X=e_{1}, Y=e_{4}, Z=e_{5}$ respectively from the equation (2).

- There is no non-zero parallel vector field on $g_{4}$.

If a non-zero vector field $\xi=\sum a_{i} e_{i}$ is parallel $(\nabla \xi=0)$, by calculating $g\left(\nabla_{e_{i}} \xi, e_{j}\right)$ for basis elements, we get $a_{i}=0$, for $i=1, \cdots, 5$. It also shows that $\nabla \eta \neq 0$ for any almost contact metric structure $(\phi, \xi, \eta, g)$ on $g_{4}$. In particular, $(\phi, \xi, \eta, g)$ is neither $\mathcal{C}_{1}$ (nearly-K-cosymplectic), nor $\mathcal{C}_{2}$.

- A vector field $\xi$ on $g_{4}$ is Killing if and only if $\xi \in\left\langle e_{5}\right\rangle$.

Let $\xi=\sum a_{i} e_{i}$ be a non-zero Killing vector field. Then, for any $e_{i}, e_{j}$, we have $g\left(\nabla_{e_{i}} \xi, e_{j}\right)=-g\left(\nabla_{e_{j}} \xi, e_{i}\right)$. Thus,

$$
\begin{aligned}
& g\left(\nabla_{e_{1}} \xi, e_{3}\right)=-g\left(\nabla_{e_{3}} \xi, e_{1}\right) \Rightarrow a_{2}=0, \\
& g\left(\nabla_{e_{1}} \xi, e_{4}\right)=-g\left(\nabla_{e_{4}} \xi, e_{1}\right) \Rightarrow a_{3}=0, \\
& g\left(\nabla_{e_{1}} \xi, e_{5}\right)=-g\left(\nabla_{e_{5}} \xi, e_{1}\right) \Rightarrow a_{4}=0, \\
& g\left(\nabla_{e_{2}} \xi, e_{3}\right)=-g\left(\nabla_{e_{3}} \xi, e_{2}\right) \Rightarrow a_{1}=0 .
\end{aligned}
$$

No condition is obtained for $a_{5}$. In other words, $\xi$ is Killing if and only if $\xi=a_{5} e_{5}$.

- $\quad$ There is no $\alpha$-Sasakian structure.

Let $(\phi, \xi, \eta, g)$ be an $\alpha$-Sasakian structure on $g_{4}$. Then, $\xi=e_{5}$, since it is a unit Killing vector field. On the other hand, by considering the relation $\nabla_{X} \xi=-\alpha \phi(X)$, we get $\phi\left(e_{2}\right)=-\frac{1}{2 \alpha} \nabla_{e_{2}} e_{5}=0$. However, in this case, $g\left(\phi\left(e_{2}\right), \phi\left(e_{2}\right)\right) \neq g\left(e_{2}, e_{2}\right)-\eta\left(e_{2}\right) \eta\left(e_{2}\right)$.

- $\quad$ There is no $\beta$-Kenmotsu structure.

Let $(\phi, \xi, \eta, g)$ be a $\beta$-Kenmotsu structure with fundamental two-form $\Phi=\sum b_{i j} e^{i j}, \xi=\sum a_{i} e_{i}$, 
$\eta=\sum b_{i} e^{i}$. Then, $g\left(\nabla_{e_{i}} \xi, e_{j}\right)=g\left(\nabla_{e_{j}} \xi, e_{i}\right)$ for any basis elements $e_{i}, e_{j}$, which implies that $\xi=a_{1} e_{1}+a_{2} e_{2}$ and $\eta=b_{1} e^{1}+b_{2} e^{2}$. However, after an easy calculation on the defining relation of a $\beta$-Kenmotsu structure, we get $b_{i j}=0$, for any $i, j$.

- There exists a semi cosymplectic structure.

For any two-form $\Phi=\sum b_{i j} e^{i j}$ any $X=\sum x_{i} e_{i} \in g_{4}$,

$$
\delta \Phi(X)=-\sum\left(\nabla_{e_{i}} \Phi\right)\left(e_{i}, X\right)=-\left\{x_{3} b_{12}+x_{4} b_{13}+x_{5} b_{14}\right\} .
$$

Thus $\delta \Phi(X)=0$ for any $X$ iff $b_{12}=b_{13}=b_{14}=0$. In addition, for any one-form $\eta=\sum b_{i} e^{i}$, we have

$$
\delta \eta=-\sum\left(\nabla_{e_{i}} \eta\right)\left(e_{i}\right)=-\sum g\left(\nabla_{e_{i}} \xi, e_{i}\right)=0 .
$$

Thus for example, the a.c.m.s. for which $\xi=e_{1}, \eta=e^{1}$ and $\Phi=e^{23}+e^{45}$ is semi cosymplectic.

- There exists no almost cosymplectic structure.

Since $d \eta(X, Y)=\frac{1}{2}\left\{\left(\nabla_{X} \eta\right)(Y)-\left(\nabla_{Y} \eta\right)(X)\right\}, d \eta(X, Y)=0$ iff $\left(\nabla_{X} \eta\right)(Y)=\left(\nabla_{Y} \eta\right)(X)$, or equivalently, $g\left(\nabla_{X} \xi, Y\right)=g\left(\nabla_{Y} \xi, X\right)$ for all $X, Y$ in $g_{4}$. Substituting basis elements for $X$ and $Y$ implies that $d \eta=0$ iff $\xi=a_{1} e_{1}+a_{2} e_{2}$. Any almost cosymplectic structure is almost-K-contact, thus for the fundamental form $\Phi=\sum b_{i j} e^{i j}$ of an almost cosymplectic structure, we have $\nabla_{\xi} \Phi=0$, where $\xi=a_{1} e_{1}+a_{2} e_{2} . \nabla_{\xi} \Phi\left(e_{i}, e_{j}\right)=0$ yields $\Phi=0$.

The algebra $g_{5}$ : By Kozsul's formula; the covariant derivatives of the basis elements are as follows:

$$
\begin{array}{llll}
\nabla_{e_{1}} e_{2}=\frac{1}{2} e_{4}, & \nabla_{e_{1}} e_{3}=\frac{1}{2} e_{5}, & \nabla_{e_{1}} e_{4}=-\frac{1}{2} e_{2}, & \nabla_{e_{1}} e_{5}=-\frac{1}{2} e_{3}, \\
\nabla_{e_{2}} e_{1}=-\frac{1}{2} e_{4}, & \nabla_{e_{2}} e_{4}=\frac{1}{2} e_{1}, & \nabla_{e_{3}} e_{1}=-\frac{1}{2} e_{5}, & \nabla_{e_{3}} e_{5}=\frac{1}{2} e_{1}, \\
\nabla_{e_{4}} e_{1}=-\frac{1}{2} e_{2} & \nabla_{e_{4}} e_{2}=\frac{1}{2} e_{1}, & \nabla_{e_{5}} e_{1}=-\frac{1}{2} e_{3}, & \nabla_{e_{5}} e_{3}=\frac{1}{2} e_{1}
\end{array}
$$

- There exists no cosymplectic structure.

The proof is similar to that in other algebras.

- $\quad$ There is no nearly cosymplectic structure on $g_{5}$.

Let $\Phi=\sum b_{i j} e^{i j}$ be the two-form of a nearly cosymplectic a.c.m.s. Replacing $X$ and $Y$ by basis elements, we have $\left(\nabla_{e_{i}} \Phi\right)\left(e_{i}, e_{j}\right)=0$, which shows that $b_{i j}=0$, except for $b_{23}, b_{25}, b_{34}$ and $b_{45}$. Then $\Phi=b_{23} e^{23}+b_{25} e^{25}+b_{34} e^{34}+b_{45} e^{45}$. We obtain $b_{23}=b_{25}=b_{34}=b_{45}=0$ for $X=e_{1}$, $Y=e_{2}, Z=e_{5} ; X=e_{1}, Y=e_{2}, Z=e_{3} ; X=e_{3}, Y=e_{1}, Z=e_{2}$ and $X=e_{4}, Y=e_{1}, Z=e_{3}$ respectively in the equation (2).

- There is no non-zero parallel vector field on $g_{5}$.

The proof is similar to other cases. In particular, $(\phi, \xi, \eta, g)$ is neither $\mathcal{C}_{1}$ (nearly-K-cosymplectic), nor $\mathcal{C}_{2}$.

- A vector field $\xi$ on $g_{5}$ is Killing if and only if $\xi \in\left\langle e_{4}, e_{5}\right\rangle$.

Let $\xi=\sum a_{i} e_{i}$ be a non-zero Killing vector field. Then, for any $e_{i}, e_{j}$, we have $g\left(\nabla_{e_{i}} \xi, e_{j}\right)=-g\left(\nabla_{e_{j}} \xi, e_{i}\right)$. Thus,

$$
\begin{aligned}
& g\left(\nabla_{e_{1}} \xi, e_{4}\right)=-g\left(\nabla_{e_{4}} \xi, e_{1}\right) \Rightarrow a_{2}=0, \\
& g\left(\nabla_{e_{1}} \xi, e_{5}\right)=-g\left(\nabla_{e_{5}} \xi, e_{1}\right) \Rightarrow a_{3}=0, \\
& g\left(\nabla_{e_{2}} \xi, e_{4}\right)=-g\left(\nabla_{e_{4}} \xi, e_{2}\right) \Rightarrow a_{1}=0 .
\end{aligned}
$$

No condition is obtained for $a_{4}$ and $a_{5}$. Thus, $\xi$ is Killing if and only if $\xi=a_{4} e_{4}+a_{5} e_{5}$.

- There is no $\alpha$-Sasakian structure.

Let $(\phi, \xi, \eta, g)$ be an $\alpha$-Sasakian structure on $g_{5}$. Then, $\xi=a_{4} e_{4}+a_{5} e_{5}$, where $a_{4}^{2}+a_{5}^{2}=1$ and $\eta=b_{4} e_{4}+b_{5} e_{5}$. By the relation $\nabla_{X} \xi=-\alpha \phi(X)$, we get $\phi\left(e_{2}\right)=-\frac{a_{4}}{2 \alpha} e_{1}$ and $\phi\left(e_{3}\right)=-\frac{a_{5}}{2 \alpha} e_{1}$. 
Since $g\left(\phi\left(e_{2}\right), \phi\left(e_{3}\right)\right)=g\left(e_{2}, e_{3}\right)-\eta\left(e_{2}\right) \eta\left(e_{3}\right)$, we have $a_{4} \cdot a_{5}=0$. This implies $\phi\left(e_{2}\right)=0$, or $\phi\left(e_{3}\right)=0$. Assume without loss of generality that $\phi\left(e_{2}\right)=0$. Then, $g\left(\phi\left(e_{2}\right), \phi\left(e_{2}\right)\right) \neq$ $g\left(e_{2}, e_{2}\right)-\eta\left(e_{2}\right) \eta\left(e_{2}\right)$.

- There is no $\beta$-Kenmotsu structure.

Let $(\phi, \xi, \eta, g)$ be a $\beta$-Kenmotsu structure with fundamental two-form $\Phi=\sum b_{i j} e^{i j}, \xi=\sum a_{i} e_{i}$, $\eta=\sum b_{i} e^{i}$. Then, $g\left(\nabla_{e_{i}} \xi, e_{j}\right)=g\left(\nabla_{e_{j}} \xi, e_{i}\right)$ for any basis elements $e_{i}, e_{j}$, which implies that $\xi=a_{1} e_{1}+a_{2} e_{2}+a_{3} e_{3}$ and $\eta=b_{1} e^{1}+b_{2} e^{2}+b_{3} e^{3}$. Replacing basis elements for $X, Y, Z$ in the defining relation of $\beta$-Kenmotsu structures results in $\Phi=0$. Thus, there does not exist a $\beta$-Kenmotsu structure.

- There exists a semi cosymplectic structure.

For any two-form $\Phi=\sum b_{i j} e^{i j}$ any $X=\sum x_{i} e_{i} \in g_{5}$,

$$
\delta \Phi(X)=-\sum\left(\nabla_{e_{i}} \Phi\right)\left(e_{i}, X\right)=-\left\{x_{4} b_{12}+x_{5} b_{13}\right\}
$$

Thus, $\delta \Phi(X)=0$ for any $X$ iff $b_{12}=b_{13}=0$. In addition, for any one-form $\eta=\sum b_{i} e^{i}$, we have

$$
\delta \eta=-\sum\left(\nabla_{e_{i}} \eta\right)\left(e_{i}\right)=-\sum g\left(\nabla_{e_{i}} \xi, e_{i}\right)=0 .
$$

Thus, for example, the a.c.m.s. for which $\xi=e_{5}, \eta=e^{5}$ and $\Phi=e^{14}+e^{23}$ is semi cosymplectic.

- There exists an almost cosymplectic structure.

Consider, for instance, the a.c.m.s. given by $\xi=e_{1}, \eta=e^{1}$ and $\Phi=e^{25}+e^{34}$.

The algebra $g_{6}$ : By Kozsul's formula, the covariant derivatives of the basis elements are as follows:

$$
\begin{aligned}
& \nabla_{e_{1}} e_{2}=\frac{1}{2} e_{3}, \quad \nabla_{e_{1}} e_{3}=-\frac{1}{2} e_{2}+\frac{1}{2} e_{4}, \quad \nabla_{e_{1}} e_{4}=-\frac{1}{2} e_{3}, \\
& \nabla_{e_{2}} e_{1}=-\frac{1}{2} e_{3}, \quad \nabla_{e_{2}} e_{3}=\frac{1}{2} e_{1}+\frac{1}{2} e_{5}, \quad \nabla_{e_{2}} e_{5}=-\frac{1}{2} e_{3}, \\
& \nabla_{e_{3}} e_{1}=-\frac{1}{2} e_{2}-\frac{1}{2} e_{4}, \quad \nabla_{e_{3}} e_{2}=\frac{1}{2} e_{1}-\frac{1}{2} e_{5}, \quad \nabla_{e_{3}} e_{4}=\frac{1}{2} e_{1}, \quad \nabla_{e_{3}} e_{5}=\frac{1}{2} e_{2}, \\
& \nabla_{e_{4}} e_{1}=-\frac{1}{2} e_{3}, \quad \nabla_{e_{4} e_{3}}=\frac{1}{2} e_{1}, \quad \nabla_{e_{5}} e_{2}=-\frac{1}{2} e_{3}, \quad \nabla_{e_{5}} e_{3}=\frac{1}{2} e_{2} .
\end{aligned}
$$

- $\quad$ There exists no cosymplectic structure on $g_{6}$.

It is easy to see that $\nabla \Phi=0$ if and only if $\Phi=0$, where $\Phi$ is a two-form.

- There is no nearly cosymplectic structure.

Let $\Phi=\sum b_{i j} e^{i j}$ be a two-form with the property that $\left(\nabla_{X} \Phi\right)(X, Y)=0$. Then, we obtain $\Phi=b_{15} e^{15}+b_{24} e^{24}+b_{45} e^{45}$. By considering $\Phi$ as the fundamental two-form of an almost contact metric structure $(\phi, \xi, \eta, g)$, from the condition $\phi^{2}=-I+\eta \otimes \xi$, we get $b_{15}^{2}=1$ and $b_{45}=0$. We get $b_{15}=b_{24}=0$ for $X=e_{5}, Y=e_{2}, Z=e_{3}$ and $X=e_{1}, Y=e_{3}, Z=e_{4}$ respectively in the equation (2).

- $\quad$ There is no non-zero parallel vector field on $g_{6}$.

The proof is the same as before.

- A vector field $\xi$ on $g_{6}$ is Killing if and only if $\xi \in\left\langle e_{4}, e_{5}\right\rangle$.

Let $\xi=\sum a_{i} e_{i}$ be a Killing vector field. Then, for any $e_{i}, e_{j}$, we have $g\left(\nabla_{e_{i}} \xi, e_{j}\right)=-g\left(\nabla_{e_{j}} \xi, e_{i}\right)$. Thus,

$$
\begin{aligned}
& g\left(\nabla_{e_{2}} \xi, e_{3}\right)=-g\left(\nabla_{e_{3}} \xi, e_{2}\right) \Rightarrow a_{1}=0, \\
& g\left(\nabla_{e_{1}} \xi, e_{3}\right)=-g\left(\nabla_{e_{3}} \xi, e_{1}\right) \Rightarrow a_{2}=0, \\
& g\left(\nabla_{e_{1}} \xi, e_{4}\right)=-g\left(\nabla_{e_{4}} \xi, e_{1}\right) \Rightarrow a_{3}=0 .
\end{aligned}
$$

No conditions are obtained for $a_{4}$ and $a_{5}$. 
- There exists no $\alpha$-Sasakian structure.

Let $(\phi, \xi, \eta, g)$ be an $\alpha$-Sasakian structure on $g_{6}$. Then, $\xi$ has the form $\xi=a_{4} e_{4}+a_{5} e_{5}$ and satisfies the equation $\nabla_{X} \xi=-\alpha \phi(X)$. Thus, the endomorphism can be expressed with:

$$
\phi\left(e_{1}\right)=\frac{a_{4}}{2 \alpha} e_{3}, \phi\left(e_{2}\right)=\frac{a_{5}}{2 \alpha} e_{3}, \phi\left(e_{3}\right)=-\frac{a_{5}}{2 \alpha} e_{2}, \phi\left(e_{4}\right)=0, \phi\left(e_{5}\right)=0 .
$$

From the condition $\phi^{2}=-I+\eta \otimes \xi$, we have

$$
\phi^{2}\left(e_{4}\right)=0=\left(a_{4}^{2}-1\right) e_{4}+a_{4} a_{5} e_{5} \Rightarrow a_{4}^{2}=1, a_{4} a_{5}=0
$$

and

$$
\phi^{2}\left(e_{5}\right)=0=a_{5} a_{4} e_{4}+\left(a_{5}^{2}-1\right) e_{5} \Rightarrow a_{5}^{2}=1, a_{4} a_{5}=0 .
$$

However, since $a_{5}^{2}=a_{4}^{2}=1$, the number $a_{4} a_{5}$ is non-zero.

- $\quad$ There is no $\beta$-Kenmotsu structure.

Let $(\phi, \xi, \eta, g)$ be a $\beta$-Kenmotsu structure with fundamental two-form $\Phi=\sum b_{i j} e^{i j}, \xi=\sum a_{i} e_{i}$. Then, $g\left(\nabla_{e_{i}} \xi, e_{j}\right)=g\left(\nabla_{e_{j}} \xi, e_{i}\right)$ for any basis elements $e_{i}$, $e_{j}$, which implies that $\xi=a_{1} e_{1}+a_{2} e_{2}$. However, after calculations on the defining relation, we get $\Phi=b_{12} e^{12}$. However, in this case, $\Phi \wedge \Phi=0$.

- There exists a semi cosymplectic structure.

The a.c.m.s. $(\phi, \xi, \eta, g)$ with $\xi=e_{3}, \eta=e^{3}$ and $\Phi=e^{14}+e^{25}$ is semi cosymplectic.

- There is no almost cosymplectic structure.

Obviously, $d e^{1}=0, d e^{2}=0, d e^{3}=-e^{12}, d e^{4}=-e^{13}, d e^{5}=-e^{23}$. Thus, for a one-form $\eta=\sum b_{i} e^{i}$, we have $d \eta=0 \Longleftrightarrow b_{3}=b_{4}=b_{5}=0$, and for a two-form $\Phi=\sum b_{i j} e^{i j}$, we get $d \Phi=0 \Longleftrightarrow b_{15}=b_{24}, b_{34}=b_{35}=b_{45}=0$. So, if $(\phi, \xi, \eta, g)$ with the fundamental two-form $\Phi=\sum b_{i j} e^{i j}$ is an almost cosymplectic structure on $g_{6}$, then, $\Phi$ and $\eta$ have the forms $\Phi=b_{12} e^{12}+b_{13} e^{13}+b_{14} e^{14}+b_{15} e^{15}+b_{23} e^{23}+b_{15} e^{24}+b_{25} e^{25}$ and $\eta=b_{1} e^{1}+b_{2} e^{2}$. However, it is easy to see that $\eta \wedge \Phi \wedge \Phi$ vanishes. Thus, the structure is not almost cosymplectic.

In summary, we state the following.

Theorem 1. An almost contact metric structure on a five-dimensional nilpotent Lie algebra $g$ is cosymplectic if and only if $g$ is abelian.

The existence of cosymplectic structures on Lie groups and on their compact quotients by uniform discrete subgroups was studied in [14]. We state Theorem 1 by direct calculation.

In the sequel, we deduce

Corollary 2. There is no cosymplectic left invariant almost contact metric structure on a five-dimensional connected Lie group whose corresponding Lie algebra is nilpotent.

Theorem 3. There is no nearly cosymplectic structure on any five-dimensional nilpotent Lie algebra.

Corollary 4. There is no nearly cosymplectic left invariant almost contact metric structure on a five-dimensional connected Lie group whose corresponding Lie algebra is nilpotent.

Theorem 5. There exists no non-zero parallel vector field on any five-dimensional nilpotent Lie algebra.

There are non-zero Killing vector fields on $g_{i}$ for $i \in\{1,2,3,4,5,6\}$.

Theorem 6. Let $g$ be one of $g_{1}, g_{2}, g_{3}$ or $g_{4}$. A vector field $\xi$ on $g$ is Killing if and only if $\xi \in<e_{5}>$. In addition, if $g$ is $g_{5}$ or $g_{6}$, then $\xi$ is Killing iff $\xi \in<e_{4}, e_{5}>$. 
Theorem 7. If $g$ has an $\alpha$-Sasakian structure, then $g$ is isomorphic to $g_{1}$.

Theorem 8. There is no $\beta$-Kenmotsu a.c.m.s. on any five-dimensional nilpotent Lie algebra.

We may conclude

Corollary 9. There is no $\beta$-Kenmotsu left invariant almost contact metric structure on a five-dimensional connected Lie group whose corresponding Lie algebra is nilpotent.

Theorem 10. There exist semi cosymplectic a.c.m. structures on each $g_{i}$.

Theorem 11. An a.c.m.s. on $g$ is almost cosymplectic iff $g$ is isomorphic to one of $g_{2}, g_{3}$ or $g_{5}$.

Let $G$ be a simply-connected nilpotent Lie group with Lie algebra $g$. It is known that there exists a co-compact discrete subgroup $\Gamma$ of $G$ such that $G / \Gamma$ is a compact nilmanifold [15]. Giving examples of discrete subgroups $\Gamma$ for simply-connected nilpotent Lie group $G_{i}$ with Lie algebra $g_{i}$ is an ongoing study.

\section{Conclusions}

In this paper, we examined almost contact metric structures on five dimensional nilpotent Lie algebras by direct calculation and obtained some results about the relations between the classes of almost contact metric structures and five dimensional nilpotent Lie algebras. In addition, we got some general results on left invariant almost contact metric structures on five dimensional nilpotent Lie groups by studying their corresponding Lie algebras.

Acknowledgments: This study was supported by Anadolu University Scientific Research Projects Commission under grant No. 1605F425.

Author Contributions: All the authors contributed equally to this work. All authors read and approved the final manuscript.

Conflicts of Interest: The authors declare no conflict of interests.

\section{References}

1. Morimoto, A. On Normal Almost Contact Structures. J. Math. Soc. Jpn. 1963, 15, 420-436.

2. Andrada, A.; Fino, A.; Vezzoni, L. A Class of Sasakian 5-Manifolds. Transform. Groups 2009, 14, 493-512.

3. Calvaruso, G.; Fino, A. Five-dimensional K-contact Lie algebras. Monatsh. Math. 2012, 167, 35-59.

4. Calvaruso, G. Three-dimensional homogeneous almost contact metric structures. J. Geom. Phys. 2013, 69, 60-73.

5. Calvaruso, G.; Perrone, A. Cosymplectic and $\alpha$-Cosymplectic Lie Algebras. Available online: http://arxiv. org/abs/1601.04572 (accessed on 18 January 2016).

6. Dixmier, J. Sur les représentations unitaires des groupes de Lie nilpotentes III. Can. J. Math. 1958, 10, 321-348.

7. Chinea, D.; Gonzales, C. A classification of almost contact metric manifolds. Ann. Mat. Pura Appl. 1990, 156, 15-36.

8. Blair, D.E. Riemannian Geometry of Contact and Symplectic Manifolds; Birkhäuser: Basel, Switzerland, 2002.

9. Alexiev, V.; Ganchev, G. On the Classification of the Almost Contact Metric Manifolds. In Proceedings of the 5th Conference Union of the Bulgarian Mathematicians, Sunny Beach, Bulgaria, 2-6 April 1986; pp. 155-161.

10. Libermann, P. Sur les automorphismes infinitésimaux des structures symplectiques et des structures de contact. In Proceedings of the Colloque de Géométrie Différentielle Globale, Bruxelles, Belgium, 1958; Centre Blge Rech. Math.: Louvain, Belgium, 1959; pp. 37-59. (In French)

11. Puhle, C. Almost contact metric 5-manifolds and connections with torsion. Differ. Geom. Appl. 2012, 30, 85-106. 
12. Gong, M.P. Classification of Nilpotent Lie Algebras of Dimension 7. Ph.D. Thesis, University of Waterloo, Waterloo, ON, Canada, 1998.

13. De Graaf, W.A. Classification of 6-dimensional nilpotent Lie algebras over fields of characteristic not 2 . J. Algebra 2007, 309, 640-653.

14. Fino, A.; Vezzoni, L. Some results on cosymplectic manifolds, Geom. Dedicata 2011, 151, 41-58.

15. Malcev, A.I. On a Class of Homogeneous Spaces; American Mathematical Society: Providence, RI, USA, 1951; p. 33.

(C) 2016 by the authors; licensee MDPI, Basel, Switzerland. This article is an open access article distributed under the terms and conditions of the Creative Commons Attribution (CC-BY) license (http:/ / creativecommons.org/licenses/by/4.0/). 